\title{
The Bt-DUX: Development of a Subjective Measure of Health-Related Quality of Life in Patients Who Underwent Surgery for Lower Extremity Malignant Bone Tumor
}

\author{
W. Peter Bekkering, $\mathrm{PT}^{1}{ }^{1}$ Theodora P.M. Vliet Vlieland, $\mathrm{PhD}^{2}{ }^{2}$ Hendrik M. Koopman, $\mathrm{PhD}^{3}$ \\ Gerard R. Schaap, MD, PhD, ${ }^{4}$ H.W. Bart Schreuder, MD, PhD, ${ }^{5}$ Auke Beishuizen, $\mathrm{MD}$, PhD, ${ }^{6}$ Wim J.E. Tissing, MD, PhD, ${ }^{7}$ \\ Peter M. Hoogerbrugge, MD, $\mathrm{PhD}^{8}{ }^{8}$ Jacob K. Anninga, MD, ${ }^{9}$ and Antonie H.M. Taminiau, MD, $\mathrm{PhD}^{2}$
}

\begin{abstract}
Background. To examine the practical applicability, internal consistency, and validity of the Bt-DUX, a disease-specific Health Related Quality of Life (HRQoL) instrument. The Bt-Dux was developed to examine patients' individual values of their life after a malignant bone tumor of the lower extremity at four domains (cosmetic, social, emotional, and functional). Procedure. Patients were eligible for this cross-sectional, multicenter study if they underwent surgery for a malignant tumor of the leg in a period ranging between 12 and 60 months before the recruitment. Assessments included: Bt-DUX, Toronto Extremity Salvage Score (TESS) Short Form (SF)-36, TNO-AZL Questionnaire for Adult's Quality of Life (TAAQOL), and TNO-AZL Children's Quality of Life Questionnaire (TACQOL). Results. Seventy-two patients (35 male, 37 female), mean age 17 (SD 4) years were included. Limb sparing surgery took
\end{abstract}

place in 32 patients and ablative surgery in 40 patients. The Bt-DUX was completed in less than $5 \mathrm{~min}$ and easy to comprehend. The mean Bt-DUX score was 69.8 (SD 15.5), with Cronbach's alpha being 0.92 . Domain-total correlations ranged between 0.84 and $0.88(P<0.01)$. Correlations between Bt-DUX Total score and TESS, SF-36 Physical and Mental Component Summary scales and selected TACQOL and TAAQOL scores were statistically significant $(P<0.05)$, except for the social scale of the TACQOL. The Bt-DUX was able to discriminate between patients with higher and lower TESS scores $(P<0.05)$. Conclusion. The Bt-DUX was found to be a practical and valid instrument. Its added value compared with existing HRQoL measures needs to be further established. Pediatr Blood Cancer 2009;53:348-355. @ (c) 2009 Wiley-Liss, Inc.

Key words: children; extremity bone tumors; quality of life; validation

\section{INTRODUCTION}

Malignant bone tumors like osteosarcoma and Ewing sarcoma mostly appear during the teenage years in the long bones of the lower extremity. The survival rates for children and adolescents with lower extremity sarcoma have improved remarkably over the past decades. Several options for surgical intervention have become available, including limb salvage by allograft or endoprosthetic reconstruction, amputation as well as rotationplasty [1]. As long as wide, tumor-free tissue margins are achieved by resection, and appropriate chemotherapy is administered, patient survival and local tumor recurrence do not depend on the type of surgery [2]. As a result of the improved life expectancy, there has been a growing interest in functional outcome and Health Related Quality of Life (HRQoL) after surgery [3-10]. Most of the studies on the outcome of surgery for malignant bone tumors were mainly focused on basic daily activities or used generic instruments for QoL. In these instruments, a number of relevant issues, such as the patient's valuation of his or her life after the disease and its surgical treatment are not specifically taken into account. Since bone tumor surgery may have considerable consequences within the domains physical activity and sports, body image, and cosmetic appearance [8], it is important to include these domains in instruments concerning QoL in persons who underwent surgery for malignant bone tumors.

For this reason, a specific bone tumor version (Bt-DUX) of an existing quality of life (QoL) instrument, the DUX-25 [10-25], was developed. The disease-specific Bt-DUX is based on the generic DUX-25 questionnaire [11]. The generic DUX-25 assesses the influence of a disease on daily activities. It contains 25 items within four different domains: social, emotional, physical, and home functioning. The scoring of the items is done by abstract faces with varying expressions (smiley's), ranging from very happy to sad. The DUX-25 has been used in studies among siblings of pediatric cancer patients [14], children with celiac disease [13-15], juvenile chronic
Additional Supporting Information may be found in the online version of this article.

${ }^{1}$ Department of Physical Therapy, Leiden University Medical Center, Leiden, The Netherlands; ${ }^{2}$ Department of Orthopaedics, Leiden University Medical Center, Leiden, The Netherlands; ${ }^{3}$ Department of Medical Psychology, Leiden University Medical Center, Leiden, The Netherlands; ${ }^{4}$ Department of Orthopedics, Academic Medical Center, University of Amsterdam, Amsterdam, The Netherlands; ${ }^{5}$ Department of Orthopaedics, Radboud University Nijmegen Medical Centre, Nijmegen, The Netherlands; ' Department of Pediatric Oncology/ Hematology, Erasmus MC University Medical Center, Sophia Children's Hospital, Rotterdam, The Netherlands; ${ }^{7}$ Department of Pediatric Oncology/Hematology, Beatrix Children's Hospital, University Medical Center Groningen, University of Groningen, The Netherlands; ${ }^{8}$ Department of Paediatric Oncology/Haematology, Radboud University Nijmegen Medical Centre, Nijmegen, The Netherlands; ${ }^{9}$ Department of Pediatrics, Leiden University Medical Center, Leiden, The Netherlands

The authors of this manuscript have no financial or personal relationships with other persons or organizations that could inappropriately influence (bias) his or her actions. The first author received outside funding in support of the research from the following entities: Johanna Children Fund, Children Fund Adrian Foundation, and BIO-Children Rehabilitation Fund.

Grant sponsor: Johanna Children Fund; Grant sponsor: Children Fund Adrian Foundation; Grant sponsor: BIO-Children Rehabilitation Fund.

*Correspondence to: W. Peter Bekkering, Department of Physical Therapy, HO-Q, Leiden University Medical Center, PO Box 9600, 2300 RC Leiden, The Netherlands. E-mail: w.p.bekkering@lumc.nl

Received 17 November 2008; Accepted 31 March 2009 
arthritis [11], malignant bone tumors [10], and proved to be internally consistent and reproducible in all studies.

The purpose of the present study is to examine the practical applicability, internal consistency, and validity of the Bt-DUX for patients with a malignant bone tumor in the lower extremity.

\section{PATIENTS AND METHODS}

\section{Study Design and Patient Recruitment}

This cross-sectional study included all patients who underwent a surgical intervention due to a malignant bone tumor in the lower extremity in five university medical centers. Patients were identified through hospital records. Patients were eligible if they were aged between 8 and 25 years at the time of the selection, if the time since surgery ranged between 12 and 60 months, if the malignant bone tumor (osteosarcoma or Ewing's sarcoma) was located around the knee and the surgical intervention consisted of limb sparing or ablative surgery. The minimum duration since surgery of 12 months was chosen to minimize the impact of the medical treatment and its complications and resulting mental consequences on physical functioning and QoL. Patients were excluded if medical conditions other than the bone tumor and its treatment limited physical activities. The selection took place between 2004 and 2006. The Medical Ethics Committee approved the study and all patients and/ or their parents gave informed consent.

\section{Development of the Bt-DUX}

For the construction of the Bt-DUX, 10 questions of the DUX25 , which were considered to be relevant for patients with bone tumors, were selected. The remaining overlap with questions in other disease-specific versions of the DUX would enable comparisons with other patient populations. After interviewing 10 patients (aged between 10 and 19 years) and 4 experienced colleagues (physical therapist, psychologist, and 2 pediatric oncologists), 10 questions were added. These questions specifically addressed functional limitations and cosmetic changes as a result of the surgical intervention. All patients and colleagues were from the Leiden University Medical Center. The principle investigator (WPB) presented a draft version, whereupon a final selection of 20 questions was made by the four experts. Similar to the generic DUX-25, the scoring of the items is done by abstract faces with varying expressions/smiley's (see Supplemental Figure), ranging from very happy (score 1) to sad (score 5). The raw item scores are converted into total and domain scores, ranging from 0 to 100 , with the highest scores indicating better HRQoL.

\section{Assessment Methods}

The practical applicability of the Bt-DUX was evaluated by interviewing 20 patients after completing the questionnaire with respect to the following aspects: completion in less than $5 \mathrm{~min}$ (yes/no) and whether the questions and answer categories were easy to comprehend (yes/no). Moreover, the rate of missing answers was determined for every question.

\section{Clinical and Demographic Characteristics}

Patient's clinical and demographic data were retrieved from registries and medical records. These data comprised: age (years), sex (male/female), type of sarcoma, location and side of the tumor, and type of surgery. In addition to the Bt-DUX, all patients received the Toronto Extremity Salvage Score (TESS) [16]. Children under 16 years of age received the TNO-AZL Children's Quality of Life Questionnaire (TACQOL) [17-19] whereas patients aged 16 years and older received the TNO-AZL Questionnaire for Adult's Quality of Life (TAAQOL) [20] and the Short Form-36 (SF-36) [21]. All patients were asked to complete the questionnaires by themselves.

The SF-36 [21] is a validated, self-administered measure of HRQoL. The SF-36 evaluates eight health concepts or domains: physical functioning, role limitation due to physical problems (role-physical), bodily pain, general perception of health, vitality, social function, role limitation due to emotional problems (role-emotional), and mental health. Each scale score ranges from 0 (worst health state) to 100 (best health state). These eight health concept scales can be converted into a Physical Component Summary (PCS) and a Mental Component Summary scale (MCS), standardized to a score with a mean of 50 and a standard deviation of 10, with scores above and below 50 indicating above and below average functioning, respectively. For that purpose, we used the scores from an age- and sex-matched, normative sample, drawn from a large, random, nationwide sample of adults $(\mathrm{N}=1742$ ) from the general Dutch population [21].

The TACQOL questionnaire [17-19] was developed as a generic instrument intended for HRQoL assessment for children aged 8 16 years old and validated in a Dutch population. The TACQOL contains 57 questions, divided over seven scales of eight items each; physical complaints, motor functioning, autonomy, cognitive functioning, social functioning, negative, and positive emotions. The score ranges were $0-4$ per item and $0-100$ per scale, with higher scores representing better HRQoL. Reliability of the scales was found to be sufficient for research purposes, with Cronbach's alpha's ranging from 0.66 to 0.79 [17].

TAAQOL questionnaire [20] is a validated, generic HRQoL questionnaire for persons of 16 years and older. The TAAQOL consists of 45 questions divided into 12 scales (most of them four items each): gross motor functioning, fine motor functioning, cognition, sleep, pain, social contacts, daily activities, sex, vitality, happiness, depressive mood, and anger. The TAAQOL questionnaire measures the emotional impact of self-reported functional problems in the same way as the TACQOL does. Internal consistency of the scales was found sufficient for research purposes [20].

The TESS [16] is a validated and reliable disease-specific measure developed to evaluate physical disability in patients treated for extremity sarcoma. The self-administered questionnaire includes 30 items on activity limitations in daily life, such as restrictions in body movement, mobility, self-care, and performance of daily tasks and routine. The degree of physical disability is rated from 0 (not possible) to 5 (without any problem). The raw score is converted to a score ranging from 0 to 100 points, with higher scores indicating less functional limitations.

\section{Statistical Analysis}

Initial descriptive analyses were performed on all variables. Total and domain scores of all variables were examined to ascertain the data distribution and assess normality. Depending on the data distribution, tests for data with normal distribution (ANOVA and independent samples $t$-test) or skewed distribution (Spearman correlation coefficients) were applied. 
The Bt-Dux Total score and domain scores were compared among the patients undergoing allograft reconstruction, endoprosthetic replacement, rotationplasty, or amputation by means of analysis of variance (ANOVA).

Internal consistency of the Bt-DUX was determined by calculating Cronbach's $\alpha$ and by computing the correlation between the four domain scores and the total Bt-DUX score (domain-total correlation). A Cronbach's $\alpha$ value of 0.85 was considered as good [22]. In order to evaluate the preconceived domain structure of the Bt-DUX, an analysis of the item-domain correlation and the Cronbach's $\alpha$ of the four domains was performed.

In the absence of a gold standard for QoL and its valuation in patients with a malignant bone tumor, we determined the construct validity of the Bt-Dux by computing associations with various other disease-specific and generic measures of QoL (previously selected subscales of the TAAQOL, TACQOL, and SF-36) or daily functioning (TESS). We hypothesized that the strength of the correlations between Bt-DUX and other measures would vary between moderate (between 0.3 and 0.5 ) and large (between 0.5 and 0.7) [23-24]. Associations exceeding the 0.7 (very large) could point in the direction of overlap between the Bt-DUX and the other instruments, and thus limit its added value.

Discriminate validity was evaluated by comparing Bt-DUX values between patients with worse and better function as categorized according to the median value of the functional outcome measure (TESS score).

\section{RESULTS}

\section{Characteristics of the Patients}

Between 2003 and 2005, one hundred four patients who underwent surgery for a malignancy of the lower extremity were identified from the registries of the five participating university hospitals and fulfilled the inclusion criteria. Of these patients, 22 died before the evaluation, the physical and mental condition of one patient did not allow for participation, two patients lived abroad and four refused participation for other reasons. The questionnaires of three patients were not complete and therefore left out of the analysis. Finally, seventy-two patients were included. Table I provides their clinical demographic characteristics. With respect to the type of surgery, patients were classified into either the limb sparing or the ablative surgery group. Rotationplasty was classified as an ablative form of surgery, because of the dependency on limb prosthesis and the cosmetic consequences compared to allograft or endoprosthetic replacements.

\section{Practical Applicability and Characteristics of the Bt-DUX and Domain Scores}

All 20 patients who were interviewed about the practical applicability reported that both question and answer categories were easy to understand and all were able to complete the questionnaire in less than $5 \mathrm{~min}$. Except for the question "getting boy/girlfriend/ relationship" (missing value in six patients, $8 \%$ ), all questions were completed by all 72 patients.

Table II shows the results of the Bt-DUX, and Table III shows the results of the other measures of QoL and functional ability. Figure 1 shows the mean and median scores and the distribution of Bt-DUX total and domain scores. The mean Bt-DUX total score and its individual domain scores varied between 50.2 and 80.9. The
TABLE I. Clinical and Demographic Characteristics of 72 Patients Who Underwent Surgical Treatment for a Malignant Bone Tumor of the Lower Extremity

\begin{tabular}{|c|c|c|c|c|}
\hline & $\begin{array}{c}\text { No. of patients } \\
(\%)\end{array}$ & Mean & SD & Range \\
\hline \multicolumn{5}{|l|}{ Gender } \\
\hline Female & $37(51 \%)$ & & & \\
\hline Male & $35(49 \%)$ & & & \\
\hline \multicolumn{5}{|l|}{ Age (years) } \\
\hline At evaluation & & 17 & 4 & $8-25$ \\
\hline At surgery & & 14 & 4 & $3-24$ \\
\hline Follow-up & & 3 & 2 & $1-5$ \\
\hline \multicolumn{5}{|l|}{ Age group } \\
\hline Children & 30 & 13 & 2 & $8-16$ \\
\hline Adolescents & 42 & 20 & 3 & $16-25$ \\
\hline \multicolumn{5}{|l|}{ Type of surgery } \\
\hline Limb sparing & $32(44 \%)$ & & & \\
\hline Allograft & $22(31 \%)$ & & & \\
\hline Prosthesis & $10(14 \%)$ & & & \\
\hline Ablative & $40(55 \%)$ & & & \\
\hline Amputation & $25(35 \%)$ & & & \\
\hline Rotationplasty & $15(21 \%)$ & & & \\
\hline \multicolumn{5}{|l|}{ Morphology } \\
\hline Osteosarcoma & $58(81 \%)$ & & & \\
\hline Ewing's sarcoma & $14(19 \%)$ & & & \\
\hline \multicolumn{5}{|l|}{ Tumor location } \\
\hline Femur & $47(65 \%)$ & & & \\
\hline Tibia/fibula & $25(35 \%)$ & & & \\
\hline
\end{tabular}

Bt-DUX total and most of the domain scores were distributed normally [22], except for the score on the social domain; this score had a skewed distribution, with $21 \%$ of the patients reporting maximal appreciation. The lowest domain score was obtained for the domain physical. There were no statistical differences of the Bt-Dux Total score and domain scores among patients who underwent allograft reconstruction, endoprosthetic replacement, rotationplasty, or amputation.

The mean score of 85.3 (SD 12.2) for the TESS indicates moderate functional limitations. The mean scores of the SF-36 were 35.3 (SD 13.9) for the Physical Component and 55.8 (SD 13.6) for the Mental Component Summary scales mean TAAQOL and TACQOL domain scores varying between 62.4 and 89.9 indicate a reasonable level of HRQoL in our population.

Table IV shows the raw mean and median scores of the Bt-DUX on the different item levels. Here, the lowest appreciation scores were obtained for the items: "the amount of sports I can do," "my abilities in sports," "my stamina," "people looking at me" (median value $3=$ ordinary, range $1-5$ ) and "the things I can't do" (median value $4=$ rather sad, range $1-5$ ).

Three of the items within the social and emotional domain: "with me it's going," "making new fiends," and "about my friends" showed median scores at the highest appreciation level ( $1=$ very happy; range $1-3$ ). Fifteen of the 20 items displayed the full range of reported scores from 1 to 5 .

\section{Internal Consistency}

The internal consistency of total and domain scores of the BtDUX appeared to be good. Cronbach's $\alpha$ was 0.92 for the total Bt- 


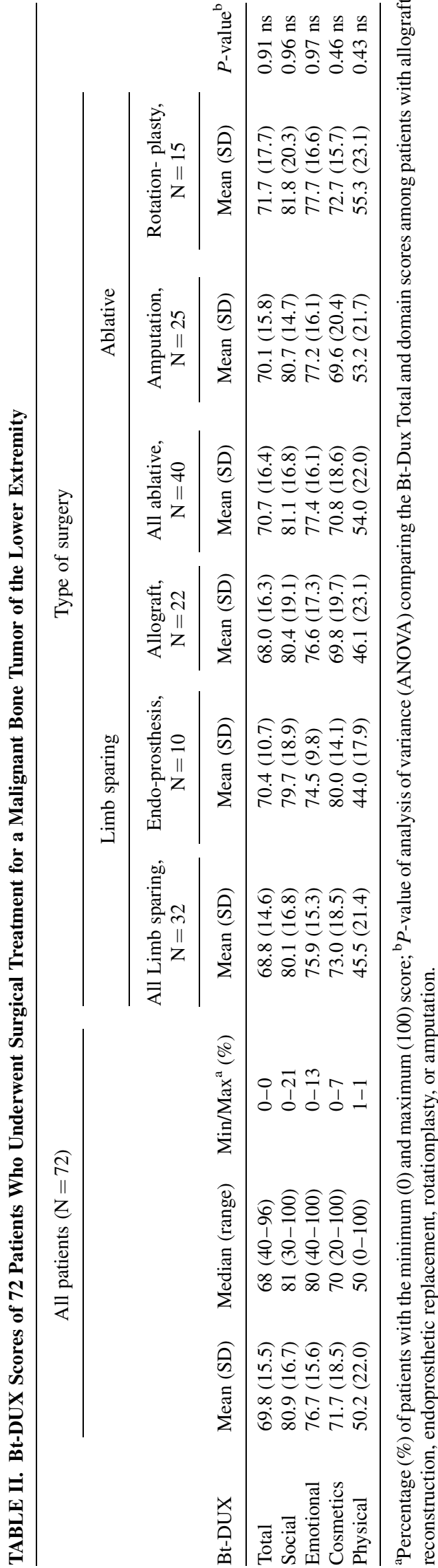

DUX, 0.73 for the emotional and cosmetics, 0.77 for the social and 0.82 for the physical function domain scores. The domain-total correlations appeared to be good, with Spearman correlation coefficients varying between 0.85 and $0.89(P<0.01)$. The itemdomain correlation was moderate, with Spearman correlation coefficients varying between 0.58 and $0.88(P<0.01)$.

\section{Construct Validity}

The results of the validity statistics are presented in Table V. The Bt-DUX demonstrates a moderate-to-strong relationship with the other measures at the domain of QoL.

The Bt-DUX total score was moderately associated with the TACQOL and TAAQOL. Spearman correlations coefficients between the DUX total score and the selected TACQOL and TAAQOL domains were statistically significant (all correlations ranging between 0.34 and $0.55, P<0.05$ ), except for the social scale of the TACQOL. The relationship between the Bt-DUX and the selected health concept scales of the SF-36 was moderate with Spearman correlation coefficients ranging between 0.41 and 0.49 $(P<0.01)$. The relationship from the Bt-DUX total score with the PCS and MCS was not statistically significant. The Bt-DUX total score was moderately related with functional limitations as estimated with the TESS (Spearman's $\mathrm{r}=0.49 ; P<0.01$ ).

The DUX-Bt domain scores correlated well with their corresponding domains of the other measures. The physical domain score was moderately to strongly associated with the motor function and autonomy scales of the TACQOL, gross motor functioning and daily activities of the TAAQOL, the PCS scale and physical functioning scale of the SF-36, and the TESS score $(\mathrm{r}=0.40-0.65$, $P<0.01)$. The emotional domain score was moderately associated with the pain and vitality scales of the TAAQOL and the MCS scale and vitality scale of the SF-36 $(r=0.40-0.53, P<0.05)$. The social domain score was moderately associated with the social scales of the TAAQOL and SF-36 $(\mathrm{r}=0.34-0.49, P<0.05)$, but showed no significant relationship with the social domain of the TACQOL. The cosmetic domain score of the DUX-Bt has no comparable score in the other measures.

\section{Discriminant Validity}

Table VI illustrates the discriminating properties of the Bt-DUX total score and the four domain scores regarding two categories of the TESS (division made according to the median TESS score). It was found that the Bt-DUX and all of its domain scores were significantly higher in the patients with a better TESS scores $(P<0.05)$.

\section{DISCUSSION}

In this cross-sectional study among patients with a malignant bone tumor of the lower extremity, it was found that a newly developed disease-specific instrument on patients' valuations of their lives, the Bt-Dux, was practically applicable, had sufficient internal consistency, and a good construct and discriminant validity.

The mean scores of the TESS, TAAQOL, TACQOL, and SF-36 as found in the present study are comparable to those found in other studies [2-10], suggesting that our population is representative for young patients' shortly after surgery due to a malignant bone tumor in the leg. The relatively high percentage of patients after ablative surgery 
TABLE III. Measures of Quality of Life and Functional Ability in 72 Patients Who Underwent Surgical Treatment for a Malignant Bone Tumor of the Lower Extremity

\begin{tabular}{clccc}
\hline & & & $\begin{array}{c}\text { Mean } \\
\text { (SD) }\end{array}$ & $\begin{array}{c}\text { Median } \\
\text { (range) }\end{array}$ \\
\hline $\begin{array}{l}\text { Health-Related Quality of life } \\
\text { TACQOL-CF }\end{array}$ & Motor & & & \\
$\quad$ Child form & Autonomy & 30 & $62.4(12.6)$ & $63(20-88)$ \\
$\quad$ Range 0-100 & Social & & $74.3(7.6)$ & $75(60-100)$ \\
TAAQOL & Gross motor functioning & 42 & $74.5(7.5)$ & $76(53-95)$ \\
Adolescent form & Pain & & $76.0(23.7)$ & $75(6-100)$ \\
Range 0-100 & Social functioning & & $89.7(22.5)$ & $81(13-100)$ \\
& Daily activities & & $76.3(25.3)$ & $88(0-100)$ \\
& Vitality & & $66.1(21.9)$ & $67(8-100)$ \\
SF-36 & PCS & 41 & $35.3(13.9)$ & $37(2-58)$ \\
Range 0-100 & MCS & & $55.8(13.6)$ & $58(9-75)$ \\
Functional Ability & & & & \\
TESS & & 72 & $85.3(12.2)$ & $89(51-100)$ \\
Range 0-100 & & & & \\
\hline
\end{tabular}

SF-36, Short Form 36; PCS, Physical Component Summary Score; MCS, Mental Component Summary Score.

could probably be explained by the young age of our patients at surgery, which makes endoprosthetic surgery less feasible, our choice to classify rotationplasty as ablative surgery, and the number of patients who underwent limb ablation as secondary surgery.

The Bt-DUX appeared to be a practically applicable instrument with easily understandable questions and answer possibilities, which could be completed in a short time notice. The question in the Bt-DUX that was most frequently left unanswered, concerned

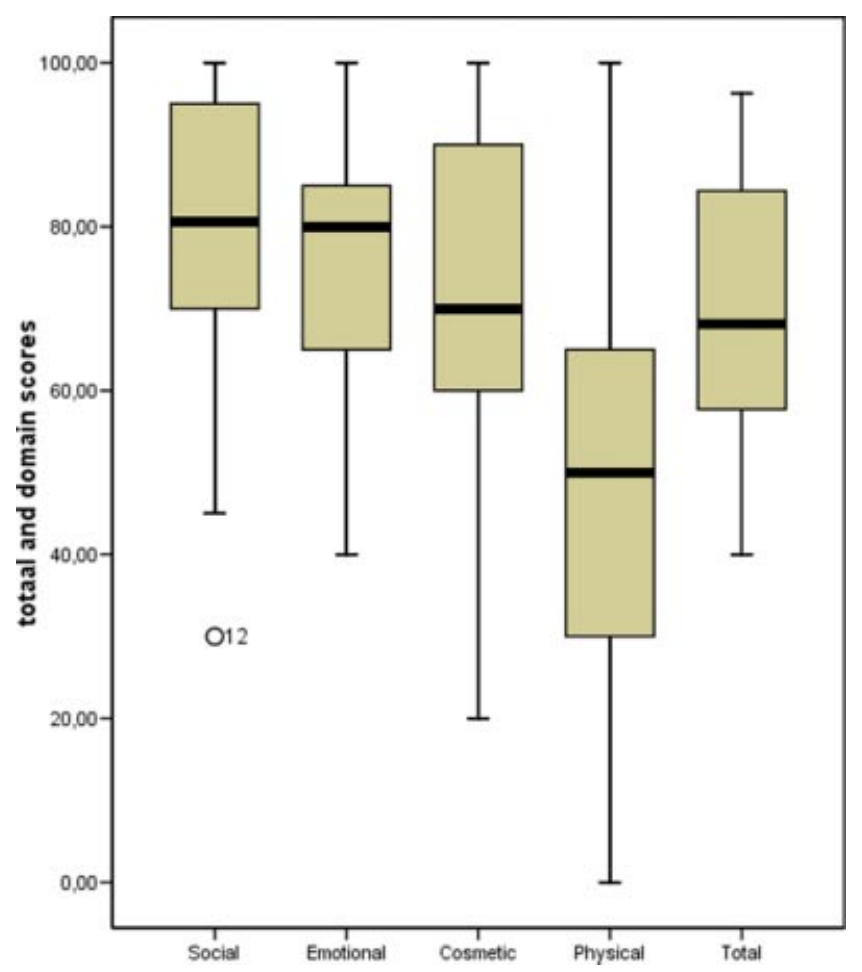

Fig. 1. Median scores and distribution of the Bt-DUX total and domain scores. [Color figure can be viewed in the online issue, which is available at www.interscience.wiley.com.] relations and/or sexuality. This low response rate could possibly be explained by the relatively low average age of the patients.

The measurement properties of the Bt-DUX appeared to be good, as the total score varying considerably and no floor or ceiling effects were seen. Moreover, the internal consistency of both the total and domain scores appeared to be sufficient [22].

In this study, the discriminating properties of the Bt-DUX were studied by comparing Bt-DUX scores between two groups of patients with different levels of physical function as classified by the TESS score, showing good discriminating properties. Discriminating properties of the Bt-DUX with respect to different surgical intervention groups still have to be determined.

The significant correlations between the Bt-DUX and measures of functional ability and selected scales for QoL and its discriminative abilities regarding patients with better or worse functional ability support the construct validity of the Bt-DUX. As the strengths of the correlations between the Bt-DUX and the other instruments within the functional ability and QoL domains were moderate to large, it is conceivable that the Bt-DUX evaluates a different aspect of QoL, namely the patients' individual values of their life after the surgical intervention, including cosmetics and sports. However, it remains to be established whether this instrument may have an additional value above the other measures commonly employed in this patient group.

The significant associations of the Bt-DUX with the other instruments for QoL and functional ability together with the discriminating properties of the instrument warrant further research into its value for use in both scientific and clinical settings. Focusing on the patient point of view is very relevant in today's society in which health care requires patients' consent and active participation [25]. The value that a person assigns to different aspects of daily living (e.g., social, emotional, physical functioning, or cosmetical contentment) influences the impact of changes due to the surgical intervention. It is generally held that ablative surgery allows patients to undertake more intensive physical activities and sports but that limb salvage is more cosmetically appealing. It is conceivable that patients differ with respect to their preferences for either sportive achievements and functional ability or the cosmetic result [8]. In the 
TABLE IV. Mean and Median Item Scores of the Bt-DUX

\begin{tabular}{|c|c|c|c|c|}
\hline Bt-DUX & Mean & Median & Range & Corresponding feeling \\
\hline \multicolumn{5}{|l|}{ Emotional } \\
\hline How it's going with me & 1.6 & 1.0 & $1-4$ & Very happy \\
\hline My life now & 1.9 & 2.0 & $1-5$ & Happy \\
\hline Other people & 2.2 & 2.0 & $1-4$ & Happy \\
\hline My health & 2.1 & 2.0 & $1-5$ & Happy \\
\hline My future & 1.9 & 2.0 & $1-5$ & Нарру \\
\hline \multicolumn{5}{|l|}{ Social } \\
\hline Getting a relationship & 1.9 & 2.0 & $1-5$ & Happy \\
\hline People at school/work & 2.0 & 2.0 & $1-5$ & Happy \\
\hline Making new (girl)friends & 1.6 & 1.0 & $1-3$ & Very happy \\
\hline The things I do & 1.8 & 2.0 & $1-4$ & Happy \\
\hline My friends & 1.6 & 1.0 & $1-3$ & Very happy \\
\hline \multicolumn{5}{|l|}{ Cosmetically } \\
\hline How my leg looks like & 1.9 & 2.0 & $1-5$ & Happy \\
\hline How my walking looks like & 2.2 & 2.0 & $1-5$ & Happy \\
\hline The way people look at me & 2.5 & 3.0 & $1-5$ & Ordinary \\
\hline My appearance/the way I look & 2.2 & 2.0 & $1-5$ & Happy \\
\hline About myself & 1.9 & 2.0 & $1-5$ & Happy \\
\hline \multicolumn{5}{|l|}{ Functional } \\
\hline My ability in sport activities & 3.2 & 3.0 & $1-5$ & Ordinary \\
\hline The amount of sport I can do & 3.1 & 3.0 & $1-5$ & Ordinary \\
\hline Abilities at school/work & 2.0 & 2.0 & $1-5$ & Happy \\
\hline My stamina & 2.9 & 3.0 & $1-5$ & Ordinary \\
\hline The things I can't do & 3.8 & 4.0 & $1-5$ & Rather sad \\
\hline
\end{tabular}

clinical setting, these preferences should be taken into account by the decision on a surgical intervention. In addition, the Bt-DUX could probably be useful to identify those patients who are strikingly unhappy with one or more aspects of their life after surgery, with the institution of subsequent interventions or support to cope with their condition and its treatment. The results of our exploratory study indicate that further research into the clinical value of the Bt-DUX is warranted.

A limitation of this study is the relatively small number of patients and the possible selection bias. Despite the fact that we included five centers, the choice for specific types of surgery may not be similar to those in other centers or countries. Due to the small

TABLE V. Spearman Correlation Coefficients Between the Domain and Total Bt-DUX Scores and Measures of Quality of Life and Functional Ability

\begin{tabular}{|c|c|c|c|c|c|}
\hline & \multicolumn{5}{|c|}{ Bt-DUX } \\
\hline & Total & Social & Emot. & Cosm. & Physic. \\
\hline \multicolumn{6}{|l|}{ TACQOL-CF } \\
\hline Motor functioning & $0.40 *$ & 0.26 & 0.35 & 0.29 & $\underline{0.48}^{* *}$ \\
\hline Autonomy & $0.55 * *$ & $\underline{0.49}^{* *}$ & $0.43 *$ & $0.41 *$ & $\overline{\mathbf{0 . 5 0}}^{* *}$ \\
\hline Social & 0.14 & $\underline{0.11}$ & 0.12 & 0.14 & 0.18 \\
\hline \multicolumn{6}{|l|}{ TAAQOL } \\
\hline Gross motor & $0.55^{* *}$ & $0.38 *$ & $0.38 *$ & $0.50 * *$ & $\underline{\mathbf{0 . 6 5}}^{* *}$ \\
\hline Pain & $0.48 * *$ & 0.30 & $\underline{0.53}^{* *}$ & $0.33 *$ & $\overline{0.49} * *$ \\
\hline Social & $0.34 *$ & $\underline{0.34} *$ & $\overline{0.24}$ & $0.35 *$ & 0.23 \\
\hline Daily activities & $0.55^{* *}$ & $0.46^{* *}$ & $0.48 * *$ & $0.43 * *$ & $\underline{\mathbf{0 . 5 3}}^{* *}$ \\
\hline Vitality & $0.46 * *$ & 0.29 & $\underline{0.45}^{* *}$ & $0.48 * *$ & $\overline{0.42} * *$ \\
\hline \multicolumn{6}{|l|}{ SF-36 } \\
\hline Physical functioning & $0.41 * *$ & 0.29 & 0.31 & $0.40 *$ & $\underline{0.52}^{* *}$ \\
\hline Social functioning & $0.49 * *$ & $\underline{0.49}^{* *}$ & $0.49 * *$ & 0.31 & $\overline{0.36} *$ \\
\hline Vitality & $0.47 * *$ & $\overline{0.41} * *$ & $\underline{0.47}^{* *}$ & $0.45 * *$ & $0.36^{*}$ \\
\hline General health & $\underline{\mathbf{0 . 4 3}}^{* *}$ & 0.27 & $\overline{0.54} * *$ & $0.47 * *$ & $0.43 * *$ \\
\hline PCS & 0.26 & 0.14 & 0.22 & 0.23 & $\underline{0.40}^{* *}$ \\
\hline MCS & 0.25 & 0.21 & $\underline{0.40}^{*}$ & 0.10 & $\overline{0.14}$ \\
\hline TESS & $0.49 * *$ & $0.33^{* *}$ & $0.43 * *$ & $0.34 * *$ & $\underline{\mathbf{0 . 5 9}} * *$ \\
\hline
\end{tabular}

PCS, Physical Component Summary Score; MCS, Mental Component Summary Score; underlined and bold, association expected between these domain scores. *Correlation is significant at the 0.05 level; **Correlation is significant at the 0.01 level. 
TABLE VI. Mean Scores (SD) of the Bt-DUX Total and Domains in Groups of Patients With Worse and Better Functional (TESS) Status

\begin{tabular}{lccc}
\hline & \multicolumn{2}{c}{ TESS scores } \\
\cline { 2 - 3 } & $\begin{array}{c}\text { Worse functional status } \\
(\text { TESS } \leq 88), \mathrm{N}=35\end{array}$ & $\begin{array}{c}\text { Better functional status } \\
(\text { TESS }>88), \mathrm{N}=37\end{array}$ & $P$-value \\
\hline Total Bt-DUX & $62.7(14.0)$ & $76.6(13.9)$ & $0.000^{* *}$ \\
Social & $76.1(16.3)$ & $85.5(15.9)$ & $0.015^{*}$ \\
Emotional & $71.0(14.8)$ & $82.2(14.6)$ & $0.002^{* *}$ \\
Cosmetics & $65.4(19.3)$ & $77.7(15.7)$ & $0.004^{* * *}$ \\
Physical & $38.4(18.7)$ & $61.4(19.0)$ & $0.000^{* *}$ \\
\hline
\end{tabular}

${ }^{*} P<0.05,{ }^{* *} P<0.01$ differences between worse and better functional status (independent samples $t$-test); worse and better functional status corresponds with less than or greater than the median value (88) of the TESS.

sample size and the cross-sectional design of the study no estimation of the effect of maturation can be made. Patients with a malignant bone tumor may start as early teens and turn into adults during and after the initial treatment. During this dynamic time in development, ideas, goals, and responses may typically change. Moreover, the ability of the instrument to show differences between different surgical interventions groups or tibia versus femur lesions, and its validity in patients in various stages of the disease, including the first year where surgical and drug treatment is intensive and may lead to complications, need to be further determined. To study these factors in greater detail, a large population and a prospective study design, preferably including multiple centers from different countries, is needed. These limitations indicate that the validity of the Bt-DUX in other populations and in other languages (e.g., English) still needs to be established.

In summary, the Bt-DUX is a practical and valid instrument measuring patients' individual QoL values after surgery due to a malignant bone tumor in the leg. Its added value with respect to its clinical and scientific usage needs to be further established.

\section{ACKNOWLEDGMENT}

Normative data SF 36 were obtained from the Division of Psycho-Social Research and Epidemiology (PSOE), The Netherlands Cancer Institute-Antoni van Leeuwenhoek Hospital.

\section{REFERENCES}

1. Nagarajan R, Neglia JP, Clohisy DR, et al. Limb salvage and amputation in survivors of pediatric lower-extremity bone tumours: What are the long-term implications? J Clin Oncol 2002;20:44934501.

2. Rougraff BT, Simon MA, Kneisl JS, et al. Limb salvage compared with amputation for osteosarcoma of the distal end of the femur. A long term oncological, functional and quality-of-life study. J Bone Joint Surg Am 1994;76:649-656.

3. Nagarajan R, Clohisy DR, Neglia JP, et al. Function and quality-oflife of survivors of pelvic and lower extremity osteosarcoma and Ewing's sarcoma: The Childhood Cancer Survivor Study. Br J Cancer 2004;91:1858-1865.

4. Akahane T, Shimizu T, Isobe K, et al. Evaluation of postoperative general quality of life for patients with osteosarcoma around the knee joint. J Pediatr Orthop B 2007;16:269-272.
5. Frances JM, Morris CD, Arkader A, et al. What is quality of life in children with bone sarcoma? Clin Orthop Relat Res 2007;459:3439.

6. Tabone MD, Rodary C, Oberlin O, et al. Quality of life of patients treated during childhood for a bone tumor: Assessment by the Child Health Questionnaire. Pediatr Blood Cancer 2005;45:207-211.

7. Ginsberg JP, Rai SN, Carlson CA, et al. A comparative analysis of functional outcomes in adolescent and Young adults with lowerextremity bone sarcoma. Pediatr Blood Cancer 2007;49:964-969.

8. Hopyan S, Tan JW, Graham HK, et al. Function and upright time following limb salvage, amputation, and rotationplasty for pediatric sarcoma of bone. J Pediatr Orthop 2006;26:405408.

9. Aksnes LH, Hall KS, Jebsen N, et al. Young survivors of malignant bone tumours in the extremities: A comparative study of quality of life, fatigue and mental distress. Support Care Cancer 2007;15: 1087-1096.

10. Koopman HM, Koetsier JA, Taminiau AH, et al. Health-related quality of life and coping strategies of children after treatment of a malignant bone tumor: A 5-year follow-up study. Pediatr Blood Cancer 2005;45:694-699.

11. Koopman HM, Kamphuis RP, Verrips GH, et al. Health related quality of life of children with juvenile chronic arthritis. Qual life Res 1997;6:196.

12. Koopman HM, Theunissen NCM, Vogels TGC, et al. The DUX-25: A short form questionnaire for measuring health related quality of life of children with chronic illness. Qual Life Res 1998;7:619.

13. Kolsteren MM, Koopman HM, Schalekamp G, et al. Health-related quality of life in children with celiac disease. J Pediatr 2001; 138:593-595.

14. Houtzager BA, Grootenhuis HN, Caron HN, et al. Quality of life and psychological adaptation in siblings of pediatric cancer patients, 2 years after diagnosis. Psycho-Oncology 2004;13:499511.

15. van Doorn RK, Winkler LM, Zwinderman KH, et al. CDDUX: A disease-specific health-related quality-of-life questionnaire for children with celiac disease. J Pediatr Gastroenterol Nutr 2008; 47:147-152.

16. Davis AM, Wright JG, Williams JI, et al. Development of a measure of physical function for patients with bone and soft tissue sarcoma. Qual Life Res 1996;5:508-516.

17. Verrips GH, Vogels AGC, Verloove-Vanhorick SP, et al. Healthrelated quality of life measure for children-The TACQOL. J Appl Ther 1997;1:357-360.

18. Verrips GH, Vogels AGC, Koopman HM, et al. Measuring healthrelated quality of life in a child population. Eur J Public Health 1999;9:188-193. 
19. Vogels T, Verrips GH, Verloove-Vanhorick SP, et al. Measuring health-related quality of life in children: The development of the TACQOL parent form. Qual Life Res 1998;7:457-465.

20. Fekkes M, Kamphuis RP, Ottenkamp J, et al. Health-related quality of life in young adults with minor congenital heart disease. Psychol Health 2001;16:239-251.

21. Aaronson NK, Muller M, Cohen PD, et al. Translation, validation, and norming of the Dutch language version of the SF-36 Health Survey in community and chronic disease populations. J Clin Epidemiol 1998;51:1055-1068.
22. Terwee CB, Bot SDM, de Boer MR, et al. Quality criteria were proposed for measurement properties of health status questionnaires. J Clin Epidemiol 2007;60:34-42.

23. Cohen J. Statistical power analysis for the behavioral sciences, 2nd edition. New Jersey: Lawrence Erlbaum; 1998.

24. Hopkins WG. Measures of reliability in sports medicine and science. Sports Med 2000;30:1-15.

25. Kvien TK, Heiberg T. Patient perspective in outcome assessments-Perceptions or something more? J Rheumatol 2003;30: 873-876. 